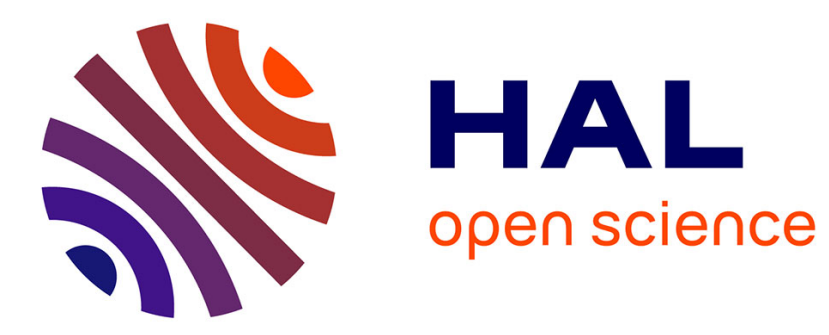

\title{
Etude de la charge engendrée par le choc d'une particule solide sur une plaque métallique
}

\author{
M. Benmadda, G. Touchard, J. Borzeix
}

\section{To cite this version:}

M. Benmadda, G. Touchard, J. Borzeix. Etude de la charge engendrée par le choc d'une particule solide sur une plaque métallique. Revue de Physique Appliquée, 1987, 22 (9), pp.1071-1074. 10.1051/rphysap:019870022090107100 . jpa-00245645

\section{HAL Id: jpa-00245645 https://hal.science/jpa-00245645}

Submitted on 1 Jan 1987

HAL is a multi-disciplinary open access archive for the deposit and dissemination of scientific research documents, whether they are published or not. The documents may come from teaching and research institutions in France or abroad, or from public or private research centers.
L'archive ouverte pluridisciplinaire HAL, est destinée au dépôt et à la diffusion de documents scientifiques de niveau recherche, publiés ou non, émanant des établissements d'enseignement et de recherche français ou étrangers, des laboratoires publics ou privés. 
Classification

Physics Abstracts

$47.60-66.30 \mathrm{~J}-73.30-73.40 \mathrm{~B}$

\title{
Etude de la charge engendrée par le choc d'une particule solide sur une plaque métallique
}

\author{
M. Benmadda, G. Touchard et J. Borzeix \\ L.E.A.-U.A. 191 du C.N.R.S., Laboratoire de Physique et Mécanique des Fluides, \\ 40, avenue du Recteur Pineau, 86022 Poitiers, France
}

(Reçu le 24 novembre 1986, révisé le 23 janvier 1987, accepté le 12 février 1987)

\begin{abstract}
Résumé. - Cette étude s'inscrit dans le cadre plus général de l'électrisation par écoulement de gaz empoussiéré à l'intérieur de conduites métalliques ou isolantes. Nous abordons ici un des points essentiels de ce phénomène, à savoir le transfert de charge au moment du choc entre les particules et la paroi interne du tube. Un dispositif expérimental, permettant de déterminer la charge d'impact d'une particule en fonction de sa charge initiale et de l'angle de choc, est tout d'abord présenté. Nous déterminons ensuite, les équations du mouvement de la particule, ce qui permet de calculer la charge initiale en fonction de la trajectoire. De l'analyse des expériences réalisées pour différents diamètres de particules sphériques, nous dégageons l'évolution de la charge d'impact en fonction de plusieurs paramètres.
\end{abstract}

\begin{abstract}
This study deals with the process of static electrification by flows of dusty gas through metallic or insulating pipes. As it is mainly controlled by the charge transfer during the shock of a particle with the inner wall of the tube we analyse on this paper such phenomenon. An experimental equipment is presented which has been perfected in order to settle the evolution of the impact charge in terms of the initial charge and the angle of the shock. The initial charge of the particle is computed through the motion equations. The experiments made for spherical particles of different diameters give the evolution of the impact charge in terms of several parameters.
\end{abstract}

\section{Introduction.}

Le phénomène de génération de charges électriques dans des écoulements de gaz empoussiéré [1] peut induire des potentiels électrique élevés sur certaines parties d'installations industrielles [2], et est par conséquent, particulièrement préoccupant, notamment en ce qui concerne les réseaux de distribution de gaz naturel.

C'est pourquoi différentes études ont été entreprises afin de mieux comprendre ce phénomène [3]. Une analyse complète de ces processus fait ressortir la nécessité d'une étude systématique des mécanismes de transfert de charges lors du contact brusque entre une particule sphérique de nature parfaitement définie et une paroi solide. Les études récentes $[4,5]$ s'accordent à montrer que l'électrisation est due principalement à la déformation de la surface de contact au moment du choc et qu'elle dépend de certains paramètres cinématiques, géométriques et dynamiques (tels que la vitesse du choc, l'angle d'incidence et le module d'Young des matériaux en contact) ; d'autres paramètres, comme les charges initiales des matériaux, restent encore mal analysés.

Notre objectif est de proposer une technique expérimentale permettant de mesurer la charge engendrée par l'impact de particules chargées tombant sous l'action combinée de leur poids, de la résistance de l'air et d'un champ électrique appliqué, sur une plaque métallique orientable.

Cette étude est faite dans le but d'établir la relation existant entre la charge initiale de la particule et la charge générée par l'impact.

\section{Dispositif et technique expérimentaux.}

Le dispositif expérimental, mis au point au laboratoire, est schématisé sur la figure 1.

Il comprend une pipette d'injection de particules 1 , une vanne 2 permettant de régler le débit, une alimentation haute tension 3 pour maintenir une zone $Z I$ de hauteur $h_{1}$ et comprise entre les électrodes 4 et 5 (de $2 \times 4 \mathrm{~cm}$ et distante de $1 \mathrm{~cm}$ ) sous champ électrique constant : $25 \times 10^{4} \mathrm{~V} / \mathrm{m}$; une élec- 


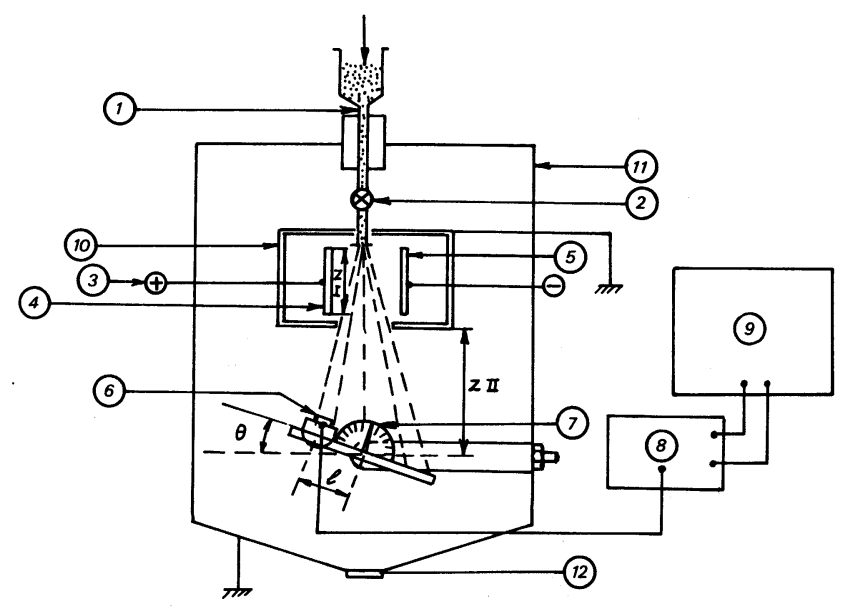

Fig. 1. - Schéma du dispositif expérimental.

[Diagram of the experimental apparatus.]

trode cible 6 , dont on peut mesurer l'inclinaison 7 , un picoampèremètre 8 relié à un enregistreur 9 et un système de vidange 12 . L'ensemble est blindé électriquement 10 et 11 . Dans son mouvement, le flux de particules heurte l'électrode 6 en donnant naissance à un courant $I$.

La quantité de charge par unité de masse correspondante est :

$$
q_{i}=I / D
$$

où $D$ désigne le débit massique des particules ayant contribué à la génération du courant $I$.

Après le choc, le flux de particules garde une charge totale par masse égale à sa charge initiale augmentée d'une charge égale et opposée à $q_{i}$

$$
q_{\mathrm{T}}=q_{0}-q_{i} .
$$

La charge d'origine $q_{0}$ peut être calculée théoriquement à partir des lois du mouvement, des données expérimentales et des coordonnées du point d'impact dans un repère $(0, X, Y)$ convenablement choisi (Fig. 2).

Si l'on désigne par $m, k, E$ et $t_{1}$, respectivement la masse de la particule, le coefficient de traînée, le champ électrique et le temps pour traverser la zone $Z I$, la charge initiale par unité de masse sera donnée par l'équation (3)

$$
q_{0} / m=\log \eta /\left(k E t_{1}^{2}\right) .
$$

Si l'on désigne par $t_{2}$ le temps pour traverser la zone $Z I I, \eta, t_{2}$ et $t_{1}$ sont obtenus à partir du système d'équations (4) [6] :

$$
\begin{aligned}
t_{1}=-\frac{1}{\sqrt{g k}} \log \left[\left(\frac{1}{2}-\frac{1}{2 c}\right) \mathrm{e}^{k h_{1}}+\right. & \\
& \left.+\left(\frac{1}{2 c}-\frac{1}{2}\right)^{2} \mathrm{e}^{2 k h_{1}}+\frac{1}{c}\right]
\end{aligned}
$$

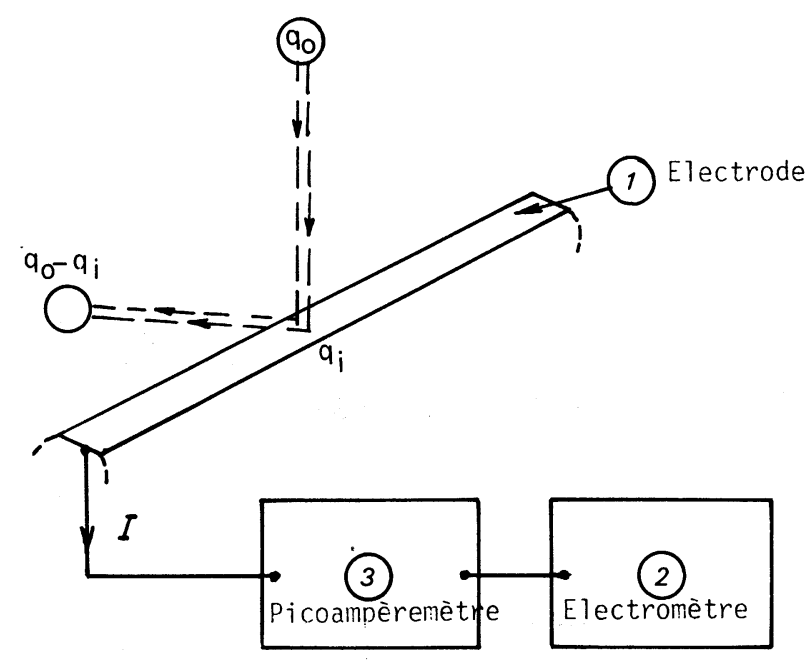

Fig. 2. - Mesure de la charge d'impact.

[Impact charge measurement.]

$$
\begin{aligned}
t_{2}= & \frac{-1}{\sqrt{g k}} \log \left[\left(\frac{1}{2}-\frac{1}{2 c}\right) \mathrm{e}^{k\left(h_{2}-\ell \sin \theta\right)}+\right. \\
& \left.+\left(\frac{1}{2 c}-\frac{1}{2}\right)^{2} \mathrm{e}^{2 k\left(h_{2}-\ell \sin \theta\right)}+\frac{1}{c}\right] \\
& \left(\eta^{2}-1\right) \frac{t_{2}}{t_{1}} \log (\eta)+1+\eta^{2}-2 \mathrm{e}^{\ell k \sin \theta}=0
\end{aligned}
$$

avec $c=\left(V_{0}-\sqrt{g / k}\right) /\left(V_{0}+\sqrt{g / k}\right), V_{0}$ étant la vitesse des particules à la sortie de la pipette $h_{1}$ la hauteur de la zone $Z I$ et $h_{2}$ la hauteur de la zone $Z I I$. Enfin $\ell$ désigne la distance entre l'électrode et son axe de rotation et $\theta$ l'angle du plan de l'électrode avec l'horizontale. Le nombre de Reynolds variant peu pendant le trajet d'une particule, le coefficient de traînée $k$ a été considéré comme constant pour chaque taille de particules. Ces équations sont résolues numériquement sur les ordinateurs du C.I.R.C.E. à Orsay.

\section{Les résultats expérimentaux.}

Nous reproduisons dans cette étude, les résultats obtenus pour quatre échantillons de particules sphériques de silice (de dimensions moyennes : 200, 280, 350 et $450 \mu \mathrm{m}$ ) pour six inclinaisons de l'électrode $\left(0^{\circ}, 5^{\circ}, 10^{\circ}, 15^{\circ}, 20^{\circ}\right.$ et $\left.25^{\circ}\right)$. La vitesse moyenne d'injection des particules est voisine de $2 \mathrm{~m} / \mathrm{s}$. Ces expériences ont été réalisées en deux étapes :

- dans un premier temps, les particules heurtent l'électrode 6 sans rester sur celle-ci, ce qui permet la mesure du courant $I$;

- dans un deuxième temps, l'électrode 6 est remplacée par un collecteur pour mesurer le débit massique des particules (dans les mêmes conditions électriques et cinématiques). 


\section{Evolution de la charge d'impact.}

Le traitement sur ordinateur (au C.I.R.C.E.) des équations (3) et (4) a permis de déterminer les charges d'origine pour chaque échantillon de particules. Les résultats obtenus sont représentés sur la figure 3 en fonction de la dimension moyenne des particules. Nous voyons que la charge par unité de masse est négative, croissante en valeur absolue en fonction de la dimension moyenne, $-7,17 \times$ $10^{-9} \mathrm{C} / \mathrm{g}$ pour les petites particules $(200 \mu \mathrm{m})$, et $-7,77 \times 10^{-9} \mathrm{C} / \mathrm{g}$ pour les grandes $(450 \mu \mathrm{m})$.

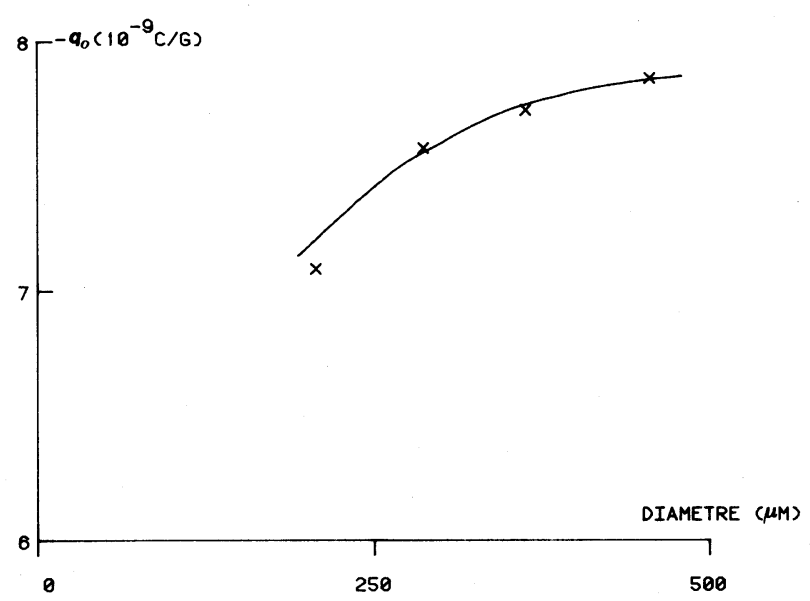

Fig. 3. - Densité de charge d'origine en fonction du diamètre de la particule.

[Origin charge density in terms of the particles diameter.]

L'évolution de la charge d'impact pour les 4 échantillons de particules est représentée sur les figures 4,5 et 6 .

La figure 4 montre que la densité massique de charge générée est de signe positif pour toutes les particules. Elle est généralement fortement décrois-

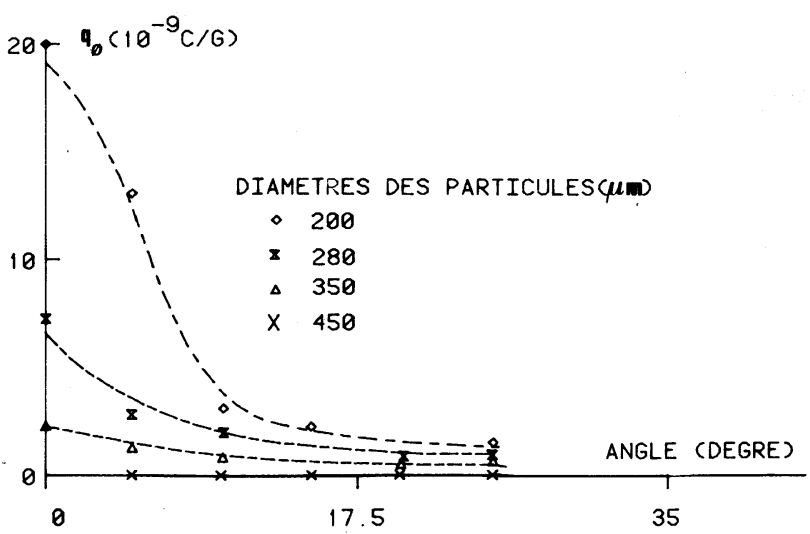

Fig. 4. - Densité de charge d'impact en fonction de l'angle de choc.

[Impact charge density in terms of the shock angle.] sante en fonction de l'angle d'incidence et ne semble pas trop influencée par celui-ci pour les particules de grande dimension $(450 \mu \mathrm{m})$. Toutes les courbes tendent vers des valeurs asymptotiques quand l'angle devient supérieur à $15^{\circ}$. Nous constatons également que la densité massique de charge obtenue avec des petits angles $\left(\theta \leqq 10^{\circ}\right)$ est généralement importante. Ceci est dû vraisemblablement à une plus grande déformation au niveau des surfaces de contact. En effet, dans ce cas les particules arrivent presque orthogonalement au plan de l'électrode et par conséquent, la majeure partie de l'énergie cinétique sert à la déformation de la surface de contact. Ceci est également confirmé par l'observation des trajectoires des particules.

La figure 5 montre l'évolution de la densité massique de charge d'impact en fonction de la

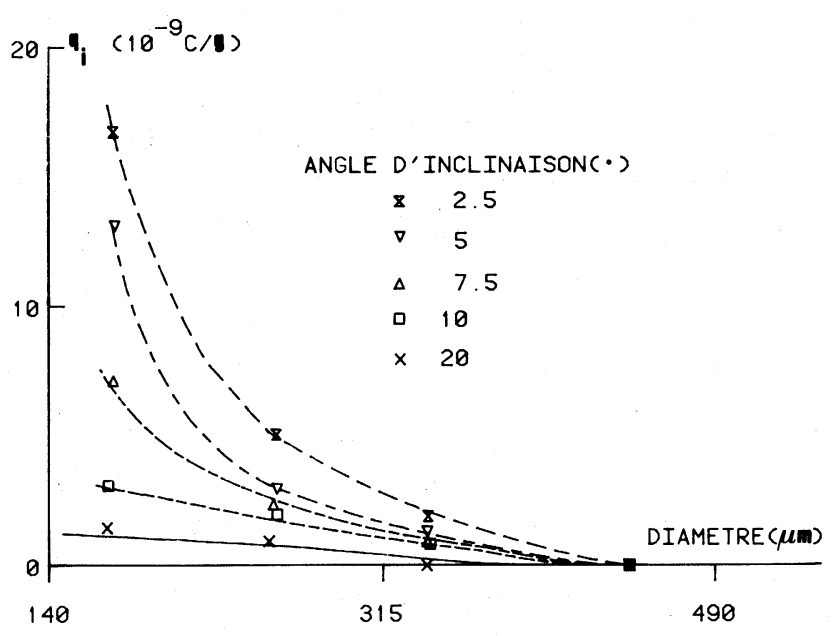

Fig. 5. - Densité de charge d'impact en fonction du diamètre de la particule.

[Impact charge density in terms of the particle diameter.]

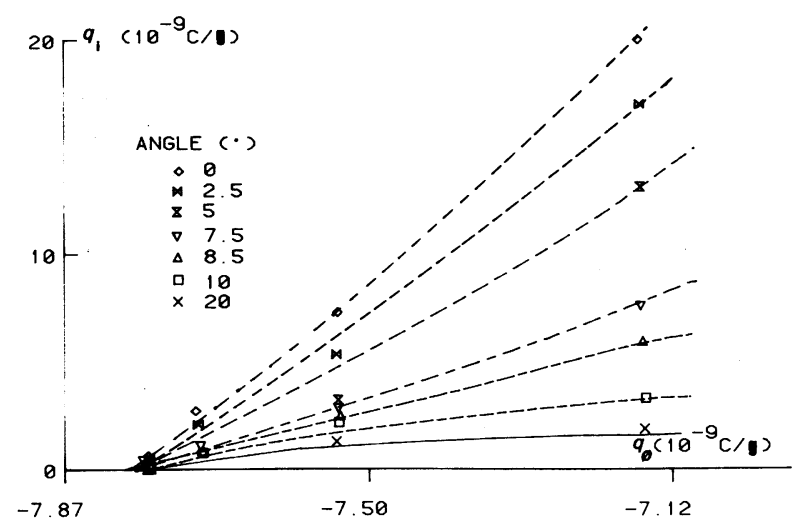

Fig. 6. - Densité de charge d'impact en fonction de la densité de charge d'origine.

[Impact charge density in terms of origin charge density.] 
dimension moyenne des particules. La charge est fortement décroissante en fonction du diamètre moyen des particules pour les faibles inclinaisons $\left(\theta \leqq 10^{\circ}\right)$. Les courbes sont presque linéaires quand l'angle devient grand $\left(\theta>15^{\circ}\right)$.

Les résultats de ces expériences nous ont permis ensuite de représenter l'évolution de la densité de charge d'impact $\left(q_{i}\right)$ en fonction de la densité de charge d'origine $\left(q_{0}\right)$. La figure 6 montre que la charge d'impact est du même ordre de grandeur que la charge d'origine et qu'elle est fortement croissante en fonction de la charge d'origine pour de faibles angles $\left(\theta<10^{\circ}\right)$. Par ailleurs toutes les courbes convergent vers un point, ce qui laisse penser qu'il y a une charge d'origine "critique " $\left(q_{0 c} \approx-7,75 \times\right.$ $10^{-9} \mathrm{C} / \mathrm{g}$ ) pour laquelle le transfert de charge entre la particule et l'électrode est nul. Nous remarquons également que pour un angle d'incidence égal à $8^{\circ} 25$, la relation entre la charge d'impact et la charge d'origine est linéaire. Ceci nous permet de déduire que pour cet angle d'incidence, la charge d'origine d'une particule dont la charge d'impact serait nulle, est environ égale à $68 \times 10^{-9} \mathrm{C} / \mathrm{g}$. Cette charge étant dans un domaine très différent de nos expériences, il convient d'être prudent sur cette extrapolation.

\section{Conclusion.}

Dans cette étude, nous avons présenté une technique pour mesurer la charge engendrée par des particules chargées électriquement tombant sous l'action combinée de leur poids, de la résistance de l'air et d'un champ électrique appliqué entre deux plaques métalliques, dans le but d'établir l'influence de la charge initiale de la particule sur la charge engendrée par l'impact.

Les résultats expérimentaux obtenus, montrent que la charge dépend énormément de l'angle d'impact quand celui-ci est faible. La dimension de la particule semble avoir aussi une influence importante, et particulièrement pour de faibles angles d'impact.

Enfin, l'analyse des expériences montre que la charge d'impact est d'autant plus importante que la charge d'origine est différente d'une valeur critique voisine de $-7,75 \times 10^{-9} \mathrm{C} / \mathrm{g}$ pour ce type de particules.

Les résultats de l'ensemble de cette étude vont maintenant pouvoir nous servir à analyser de manière plus rigoureuse le transport de charge produit pour un écoulement de gaz empoussiéré par ce type de particules.

\section{Bibliographie}

[1] Cole, B. N., Baum, M. R. and Mobbs, F. R., Proc. Inst. Mech. Eng. 184 (1969-1970) 59-67 et 77-83.

[2] Touchard, G., Borzeix, J., SAunière, Rev. Gen. Electr. 7 et 8 (1985) 592-602.

[3] Benmadda, M., TouchaRd, G., J. Electrostatics 15 (1984) 307-314.
[4] Masuda, H., KoIChi, I., IInOya, Y., AICHE J. 24 (1978) 950-956.

[5] Coste, J., Acte des Journées d'Etudes Electrostatiques, 25-26 octobre 1984.

[6] Benmadda, M., Thèse de Doctorat, Poitiers, mai 1986. 